\title{
Chaos and ergodicity in an entangled two-qubit Bohmian system
}

\author{
A.C. Tzemos and G. Contopoulos \\ Research Center for Astronomy and Applied Mathematics of the Academy of \\ Athens - Soranou Efessiou 4, GR-11527 Athens, Greece \\ E-mail: thanasistzemos@gmail.com, gcontop@academyofathens.gr
}

\begin{abstract}
We study in detail the onset of chaos and the probability measures formed by individual Bohmian trajectories in entangled states of two-qubit systems for various degrees of entanglement. The qubit systems consist of coherent states of 1-d harmonic oscillators with irrational frequencies. In weakly entangled states chaos is manifested through the sudden jumps of the Bohmian trajectories between successive Lissajous-like figures. These jumps are succesfully interpreted by the 'nodal point-X-point complex' mechanism. In strongly entangled states, the chaotic form of the Bohmian trajectories is manifested after a short time. We then study the mixing properties of ensembles of Bohmian trajectories with initial conditions satisfying Born's rule. The trajectory points are initially distributed in two sets $S_{1}$ and $S_{2}$ with disjoint supports but they exhibit, over the course of time, abrupt mixing whenever they encounter the nodal points of the wavefunction. Then a substantial fraction of trajectory points is exchanged between $S_{1}$ and $S_{2}$, without violating Born's rule. Finally, we provide strong numerical indications that, in this system, the main effect of the entanglement is the establishment of ergodicity in the individual Bohmian trajectories as $t \rightarrow \infty$ : different initial conditions result to the same limiting distribution of trajectory points.
\end{abstract}

\section{Introduction}

Bohmian Quantum Mechanics (BQM) is an interpretation of Quantum Mechanics according to which the quantum particles follow deterministic trajectories dictated by the Bohmian equations:

$$
m_{i} \frac{d x_{i}}{d t}=\frac{\hbar}{G}\left(\frac{\partial \Psi_{I}}{\partial x_{i}} \Psi_{R}-\frac{\partial \Psi_{R}}{\partial x_{i}} \Psi_{I}\right), \quad i=1,2, \ldots,
$$


where $\Psi=\Psi_{R}+i \Psi_{I}$ is the wavefunction, guided by the usual time-dependent Schrödinger equation $\hat{H} \Psi=i \hbar \partial \Psi / \partial t$, and $G=\Psi_{R}^{2}+\Psi_{I}^{2}$ [1, 2, 3, 4, 5].

Quantum Entanglement (QE) is a special physical property which characterizes the quantum systems and has a central role in the theory of Quantum Information and Computation, both from a theoretical and a technological standpoint. The construction of quantum computing machines is based solely on the understanding and manipulation of QE [6, 7]. In addition, QE is of fundamental importance in Statistical Mechanics of quantum systems, in general, since it governs the quantum probabilities of subsystems entangled with their environments.

The highly nonlinear character of the Bohmian equations motivated several works studying the dynamics of Bohmian trajectories (see, e.g. [8, 9, 10, 11, 12, 13, 14, 15]). In our previous works we studied the existence of order and chaos in Bohmian trajectories both in 2 and 3 dimensions and proposed a general theoretical framework describing the production of chaos in BQM [16, 17, 18, 19, 20]. We also studied extreme cases where the Bohmian trajectories (both ordered and chaotic) evolve on certain integral surfaces, a phenomenon we called partial integrability [21]. Furthermore, within the framework of BQM, the existence of chaos was found to be crucial for the convergence of the trajectory probability density to Born's rule. [16, 22].

Having as a goal the exploration of chaos in the presence of QE (see also [23, 24, 25]), in a previous paper [26] we gave the basic characteristics of the Bohmian trajectories in an entangled two-qubit system, where the basic qubit states in one dimension are realized as properly engineered coherent states of the unperturbed quantum harmonic oscillator. We chose to work with this system, since it is quite well understood from a quantum mechanical standpoint and is easily constructed and controlled in the laboratory. Our study showed that this model, although simple, is characterized by very rich dynamics in the Bohmian framework. We found that the choice of the physical parameters and especially of the frequency ratio $\omega_{x} / \omega_{y}$, can lead to chaotic motion (irrational $\omega_{x} / \omega_{y}$ ), periodic motion (rational $\omega_{x} / \omega_{y}$ ) and even to integrable motion $\left(\omega_{x} / \omega_{y}=1\right)$. This rich variety of Bohmian trajectories stems from two basic characteristics of our model: i) The high complexity of the Bohmian equations which appears despite the apparent simplicity of the underlying wavefunction, and ii) the existence of an infinite number of nodal points of the wavefunction, forming a time-varying lattice structure [26]. As already shown in $[17,19,20$, chaos is introduced by the approach of a trajectory to a 'nodal point-X- 
point complex' (NPXPC). This is a geometrical structure of the Bohmian flow which appears generically in the close neighbourhood of a moving nodal point.

In the present paper we study in detail the question of how chaos affects the way the Bohmian trajectories produce a covering of the configuration space, while satisfying Born's rule $P=|\Psi|^{2}$ at any time $t$.

In the first part of the paper (Sections 2-4) we study in detail the production of chaos in the Bohmian trajectories of this system through the prism of the NPXC mechanism, by monitoring the motion of the multiple NPXPCs for different amounts of entanglement and we comment on the short term evolution of the Bohmian trajectories. In the second part (Section 5) we study the long term covering of the configuration space by the Bohmian trajectories where our main result is that, in this system, the increase of entanglement generates approximate ergodicity after a moderately long time. In particular we study numerically individual Bohmian trajectories and find that the long term distribution of the points of an individual trajectory $(x(t), y(t))$ is independent from their initial conditions $(x(0), y(0))$. The convergence to the limiting distribution (corresponding to $t \rightarrow \infty$ ) can be reached in a much shorter time if we take a collective distribution of many initial conditions satisfying initially the Born rule. Consequently the measure $D(x, y)$ formed by taking the average in time covering of the space by a single trajectory $(x(t), y(t))$ is approximately equal to the mean of the quantity $|\Psi|^{2}$. This is a remarkable property of our system, since it shows that entanglement implies not only chaos, but also ergodicity, a unique property of certain dynamical systems, which, to our knowledge, has not been addressed in Bohmian Mechanics. Finally in Section 6 we draw our conclusions and present some interesting questions for future research based on our results.

\section{Our model}

The time-dependent state $|\alpha(t)\rangle$ of a 1-d coherent state of the harmonic oscillator satisfies the eigenvalue equation

$$
\hat{\alpha}|\alpha(t)\rangle=A(t)|\alpha(t)\rangle
$$

where $\hat{\alpha}$ is the anihilation operator and $A(t)=|A(t)| \exp (i \phi(t))$ its complex eigenvalue. The wavefunction $Y$ corresponding to the state $|\alpha(t)\rangle$ in the position 
representation is given by:

$Y(x, t)=\left(\frac{m \omega}{\pi \hbar}\right)^{\frac{1}{4}} \exp \left[-\frac{m \omega}{2 \hbar}\left(x-\sqrt{\frac{2 \hbar}{m \omega}} \Re[A(t)]\right)^{2}+i\left(\sqrt{\frac{2 m \omega}{\hbar}} \Im[A(t)] x+\xi(t)\right)\right]$

with

$$
\begin{aligned}
& \Re[A(t)]=a_{0} \cos (\sigma-\omega t), \Im[A(t)]=a_{0} \sin (\sigma-\omega t) \\
& \xi(t)=\frac{1}{2}\left[a_{0}^{2} \sin (2(\omega t-\sigma))-\omega t\right],
\end{aligned}
$$

where $\sigma=\phi(0)$ is the initial phase of the complex eigenvalue $A(t)$ and $a_{0} \equiv|A(0)|$. Following our previous paper [26] we study a system of two non interacting 1-d oscillators along the $x$ and $y$ direction respectively

$$
H=\frac{p_{x}^{2}}{2 m_{x}}+\frac{p_{y}^{2}}{2 m_{y}}+\frac{1}{2} m_{x} \omega_{x}^{2} x^{2}+\frac{1}{2} m_{y} \omega_{y}^{2} y^{2} .
$$

The state of our system is described by wavefunctions of the form:

$$
\Psi(x, y, t)=c_{1} Y_{R}(x, t) Y_{L}(y, t)+c_{2} Y_{L}(x, t) Y_{R}(y, t)
$$

where

$$
\begin{aligned}
& Y_{R}(i, t) \equiv Y\left(i, t ; \omega=\omega_{i}, m=m_{i}, \sigma=\sigma_{i}\right), i=x, y \\
& Y_{L}(i, t) \equiv Y\left(i, t ; \omega=\omega_{i}, m=m_{i}, \sigma=\sigma_{i}+\pi\right), i=x, y .
\end{aligned}
$$

$Y_{R}$ and $Y_{L}$ are one-dimensional coherent states with center started to the right or to the left from the center of the oscillation along $x$ or $y$. By choosing $a_{0}=5 / 2$ the wavefunctions $Y_{R}$ and $Y_{L}$ acquire a negligible overlap in Hilbert space, and can be realised as the basis states for a qubit. Consequently the wavefunction $\Psi(x, y, t)$ refers to an entangled state of two qubits made of coherent states along the coordinates $x$ and $y$. The entanglement depends on the values of the constants $c_{1}, c_{2}$, which are chosen here to be real for the sake of simplicity with $c_{1}^{2}+c_{2}^{2}=1$. Consequently we can control the amount of entaglement by altering the value of one of these amplitudes, e.g. $c_{2}$, with $c_{2} \in[0, \sqrt{2} / 2]$, where $c_{2}=0$ corresponds to a product state and $c_{2}=\sqrt{2} / 2$ corresponds a maximally entangled state (Bell state). We note that the absence of interacting terms in our Hamiltonian guarantees the conservation of QE. 


\section{Nodal trajectories}

The positions of the nodal points of the wavefunction (77) are the solutions of the equation $\Re \Psi=\Im \Psi=0$ and evolve in time according to:

$$
\begin{aligned}
x_{\text {nod }} & =\frac{\sqrt{2}\left(k \pi \cos \left(\omega_{y} t\right)+\sin \left(\omega_{y} t\right) \ln \left(\left|\frac{c_{1}}{c_{2}}\right|\right)\right)}{4 \sqrt{\omega_{x}} a_{0} \sin \left(\omega_{x y} t\right)} \\
y_{\text {nod }} & =\frac{\sqrt{2}\left(k \pi \cos \left(\omega_{x} t\right)+\sin \left(\omega_{x} t\right) \ln \left(\left|\frac{c_{1}}{c_{2}}\right|\right)\right)}{4 \sqrt{\omega_{y}} a_{0} \sin \left(\omega_{x y} t\right)}
\end{aligned}
$$

with $k \in Z, k$ even for $c_{1} c_{2}<0$ or odd for $c_{1} c_{2}>0$ and $\omega_{x y} \equiv \omega_{x}-\omega_{y}$. We choose to work with positive $c_{1}, c_{2}, \omega_{x}=1$, and $\omega_{y}=\sqrt{3}$. Consequently the ratio $\omega_{x} / \omega_{y}$ is irrational and the $k^{\prime} s$ are odd. From Eqs. (9]10) we see that the distance between two nodal points labeled by the integers $k_{1}$ and $k_{2}$ is $d \propto\left|k_{1}-k_{2}\right|$. In particular the distance between the nodal points $k_{i}$ and $k_{i+1}$ is equal to that between $k_{i}$ and $k_{i-1}$ at every given time $t$. This implies that the nodal points form a lattice structure on the plane $(x, y)$ at any time $t$.

The trajectories of different nodal points (for many odd values of $k$ ) for some values of entanglement are shown in Fig 1, These trajectories fill the whole central part of the plane $(x, y)$ except for the white regions. We observe that even with a very small amount of entanglement $\left(c_{2}=10^{-7}\right)$ the moving nodal points cover a large part of space. This fact plays a crucial role in our analysis, as it implies that we have a significant probability of close encounters between nodal points and their surrounding nodal point-X-point complexes (NPXPC, see below) and the wandering Bohmian trajectories (see section 4 ), as $t \rightarrow \infty$. The decrease of entanglement enlarges the central empty region (the higher the density in an area the lower the velocity of the nodes in this area). Consequently, the increase of entanglement confines the "slow part" of the nodal trajectories closer to the origin. In the case of a product state $\left(c_{2}=0\right)$ we have $x_{\text {nod }}=y_{\text {nod }}=\infty$, i.e. there are no nodal points.

The study of the velocities of the nodal point is important because nodal points

with large velocities do not affect significantly the Bohmian trajectories, as pointed out in [19] (sections II C, D). 

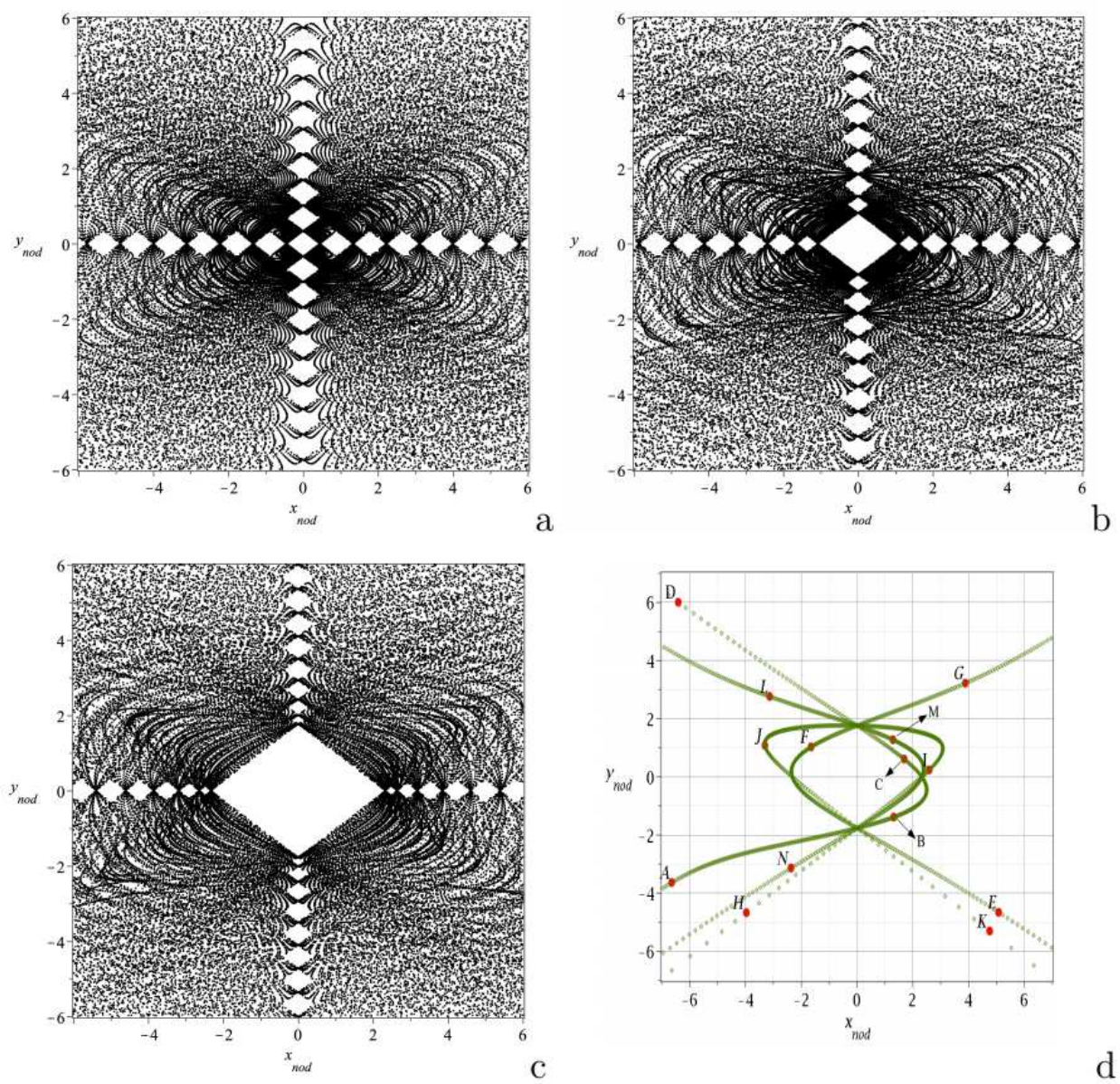

Figure 1. Nodal trajectories corresponding to the odd values of $k \in[-23,23]$ for $t \in[0,100]$ with fixed time step $t_{s}=0.01$ and different values of entanglement: (a) $c_{2}=\sqrt{2} / 2$ (maximal entanglement) (b) $c_{2}=10^{-3}$ (c) $c_{2}=10^{-7}$. The density of the trajectories in the area around the origin decreases with the decrease of the entanglement (the central empty region increases as $c_{2}$ decreases). The areas of high concentration of points are those where the velocity of the nodes is relatively small. (d) The trajectory of the nodal point $k=1$ for $t \in[0,17.2]$ when $c_{2}=10^{-7}$. (a): The nodal point starts at $x=y=-\infty$ and passes through the points A-N at the times in parentheses: $\mathrm{A}(0.34), \mathrm{B}(2.05), \mathrm{C}(3.20), \mathrm{D}(3.95), \mathrm{E}(4.80), \mathrm{F}(6.70)$, $\mathrm{G}(7.80), \mathrm{H}(8.80), \mathrm{I}(9.30), \mathrm{J}(12.00), \mathrm{K}(12.72), \mathrm{L}(13.81), \mathrm{M}(14.73), \mathrm{N}(16.50)$. Dots indicate large velocities. 


\section{Bohmian trajectories}

The Bohmian equations of motion derived by substituting the wavefunction (17) in Eqs. (1) are:

$$
\begin{aligned}
& \frac{d x}{d t}=-\frac{\sqrt{2 \omega_{x}}}{G} a_{0}\left(A \cos \left(\omega_{x} t\right)+B \sin \left(\omega_{x} t\right)\right) \\
& \frac{d y}{d t}=\frac{\sqrt{2 \omega_{y}}}{G} a_{0}\left(A \cos \left(\omega_{y} t\right)+B \sin \left(\omega_{y} t\right)\right)
\end{aligned}
$$

where

$$
\begin{aligned}
& A=2 c_{1} c_{2} \mathrm{e}^{2 f_{x}+2 f_{y}} \sin \left(2\left(g_{x}-g_{y}\right)\right), \quad B=c_{1}^{2} e^{4 f_{x}}-c_{2}^{2} e^{4 f_{y}} \\
& G=2 c_{1} c_{2} \mathrm{e}^{2 f_{x}+2 f_{y}} \cos \left(2\left(g_{x}-g_{y}\right)\right)+\mathrm{e}^{4 f_{y}} c_{2}{ }^{2}+\mathrm{e}^{4 f_{x}} c_{1}{ }^{2}
\end{aligned}
$$

with

$$
\begin{aligned}
& f_{x}=\sqrt{2 \omega_{x}} a_{0} \cos \left(\omega_{x} t\right) x, \quad f_{y}=\sqrt{2 \omega_{y}} a_{0} \cos \left(\omega_{y} t\right) y, \\
& g_{x}=\sqrt{2 \omega_{x}} a_{0} \sin \left(\omega_{x} t\right) x, \quad g_{y}=\sqrt{2 \omega_{y}} a_{0} \sin \left(\omega_{y} t\right) y
\end{aligned}
$$

and $c_{1}^{2}+c_{2}^{2}=1$.

In the case $c_{2}=0$ (product state) the system acquires the solution: $\left(x-x_{0}=\right.$ $\left.\frac{\sqrt{2 \omega_{x}}}{\omega_{x}} a_{0}\left(\cos \left(\omega_{x} t\right)-1\right), y-y_{0}=-\frac{\sqrt{2 \omega_{y}}}{\omega_{y}} a_{0}\left(\cos \left(\omega_{y} t\right)-1\right)\right)$ where $\left(x_{0}, y_{0}\right)$ are the initial conditions for $t=0$. Thus the trajectories are Lissajous figures, whose size is the same for all initial conditions $\left(|\Delta x|=\frac{2 \sqrt{2 \omega_{x}}}{\omega_{x}} a_{0} \simeq 7.1,|\Delta y|=\frac{2 \sqrt{2 \omega_{y}}}{\omega_{y}} a_{0} \simeq 5.4\right)$.

As the nodal point passes an inifinite number of times through the region occupied by the trajectories inside the support of the wavefunction of the system (the region of the configuration space in which the value $|\Psi|^{2}$ is relatively high), there is a high probability of a close interaction between the nodal points and the trajectories. We refer the reader to [17, 19, 20] on how this interaction takes place. Briefly, the nodal points form in their neighborhood a structure of the quantum flow called the 'nodal point-X-point complex' (NPXPC). The trajectories exhibit hyperbolic scattering events at each close encounter with a NPXPC, and these encounters are responsible for the chaotic behavior of the trajectories.

We calculated some trajectories starting at the same initial point $\left(x_{0}=y_{0}=1\right)$ for various values of $c_{2}$. For very small values of $c_{2}$ the trajectories are close to Lissajous curves for some time, and then the trajectories become chaotic due to a close approach to a nodal point and its associated X-point [17, 19]. It is remarkable 


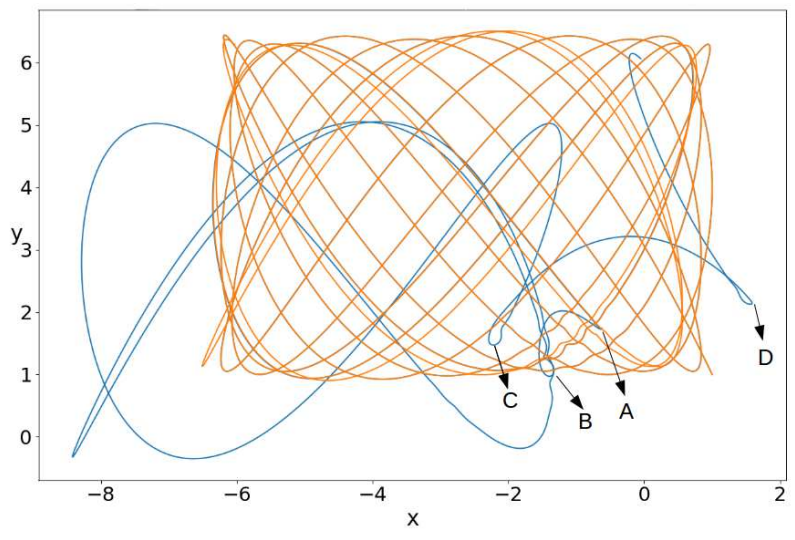

Figure 2. Two trajectories up to $t=60$, starting at $(x(0)=y(0)=1)$ with $c_{2}=1.025 \times 10^{-7}$ (red) and $c_{2}=1.03$ (blue). The two trajectories almost coincide up to $t=43.3$ (point A). After that the red orbit continues without any major perturbation, while the blue orbit has abrupt perturbations (close approaches to a NPXPC) at points $A(t=43.3), B(t=44.1), C(t=57.3)$ and $D(t=58.6)$.

that a relatively fast transition from a near-Lissajous curve (for times up to $t=1000$ ) to chaotic trajectories happens for quite small values of $c_{2}$. In fact the trajectory for $c_{2}=1.025 \times 10^{-7}$ is very similar to a Lissajous figure with $c_{2}=0$, while for $\left|c_{2}\right|=1.03 \times 10^{-7}$ the trajectory is chaotic after a time $t=43.3$ (Fig. 21), although the two trajectories almost coincide up to this time.

When $c_{2}$ is small the orbit after a close approach to a NPXPC deviates from a Lissajous curve. After close approaches to NPXPCs it tends to form several approximate Lissajous curves (Fig. [3a). After a much longer time the orbit covers the central part of the configuration space corresponding to the support of the wavefunction, as we will see in the next session. On the other hand if $c_{2}$ is large there is no clear tendency to form approximate Lissajous curves and the trajectories are quite irregular from the beginning. An example is given in Fig. Bb for the case of maximum entanglement where $c_{1}=c_{2}=\sqrt{2} / 2$.

The effect of the approach of a trajectory NPXPC can be quantified in terms of 

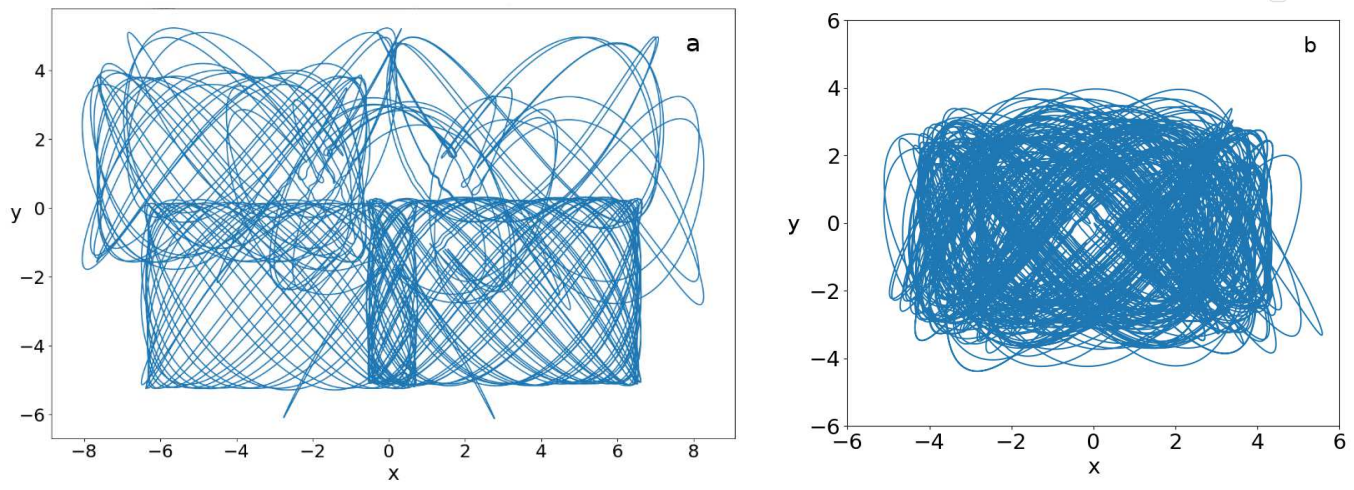

Figure 3. (a) A trajectory with $c_{2}=2 \times 10^{-5}$ and $(x(0)=-2, y(0)=2)$ for time up to $t=500$. We observe the formation of Lissajous-like figures between the encounters of the trajectory with the NPXPCs. (b) A trajectory with the same initial conditions in the maximum entangled case $c_{2}=\sqrt{2} / 2$ for a time up to $t=1000$. The motion is chaotic.

the time behavior of the value of the stretching number

$$
a_{i}=\ln \left(\frac{\xi_{i+1}}{\xi_{i}}\right)
$$

where $\xi_{i+1} / \xi_{i}$ are the infinitesimal deviations from a trajectory at two consequtive time steps $t_{i}, t_{i+1}$. In the case of the trajectory with $c_{2}=2 \times 10^{-5}$ and initial conditions $x_{0}=-2, y_{0}=2$ (Fig. 4a) the main deviations of $a_{i}$ are marked (Fig. 4 $\mathrm{b}$ ) by successive letters A-J. The corresponding times are $t=0.76,11.5 \ldots$ (as given in Fig. 4). At these times the trajectory is near the points A-J of Fig. 4a, where it exhibits some irregularity. At the points $\mathrm{A}$ and $\mathrm{J}$ the trajectory forms loops and reverses its direction. In fact at the point $J$ the trajectory undergoes large acceleration that brings it well outside the approximate Lissajous curve. One can verify that close to the other points (B-I) the trajectory shows also some small irregularities but does not form a loop. In fact, a detailed calculation shows that the loops observed at the points $\mathrm{A}$ and $\mathrm{J}$ correspond to close approaches in which the trajectory surrounds the nodal point. These are the so called 'Type I scattering events' (Fig. 2a of [19]). On the other hand the other approaches (B-I) are of type II, as seen in the same figure of [19], and do not surround the nodal point.

In the present case we have an infinity of nodal points (and their associated NPXPCs) for various values of $k$. The trajectories may change their direction by 
approaching any one of these NPXPCs and they may go around any one of the nodal points. In Fig. 5 we have drawn the velocity field in a frame centered at the nodal point $k=-1$. The other nodal points are along a line which rotates slowly around the origin, along with a gradual in time change of the nearby velocity field. The interaction between the trajectories and the nodal points is better illustrated by showing the Bohmian velocity field corresponding to the quantum flow as viewed in a frame of reference co-moving with one nodal point. Figure 5 shows the initial poisitions of six trajectory points, and their positions as time progresses are given in four snapshots. We clearly see that the trajectory points follow the velocity field and are deflected as they approach some NPXPC.

In all the approaches the distance of the moving point from a nodal point, passes through a minimum. Furthermore, near the points A-J of Fig. 4a,b the velocity of the nodal point is close to a minimum.
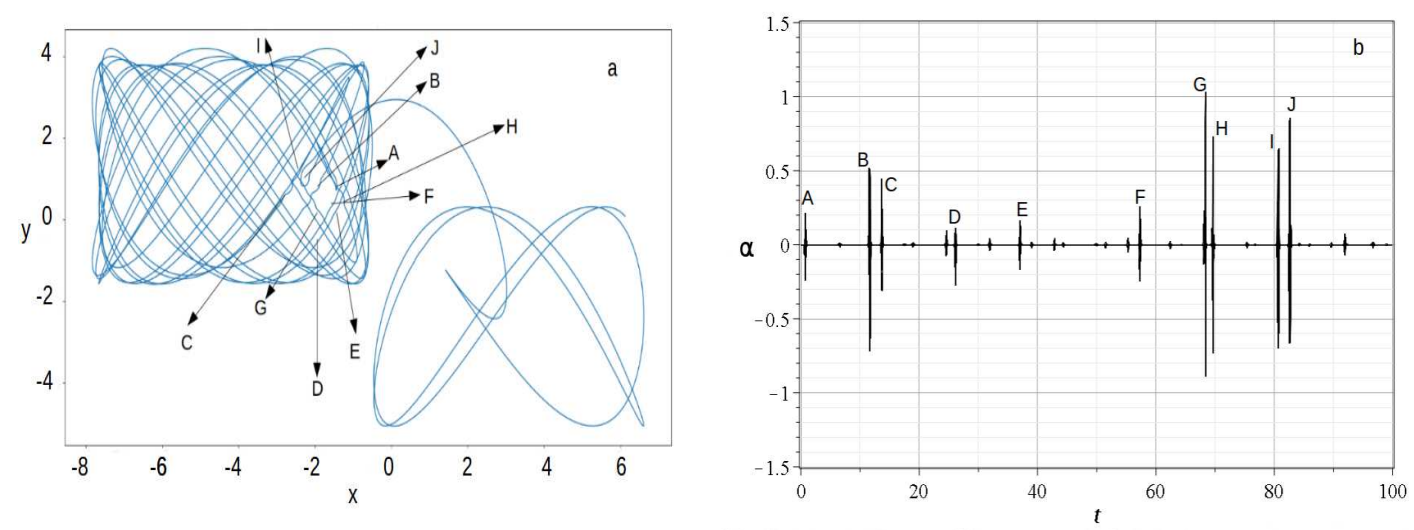

Figure 4. (a) An orbit with $c_{2}=2 \times 10^{-5}$ and initial point $(x(0)=-2, y(0)=2)$ for $t \in[0,100]$. The letters A-J indicate where the orbit approaches a nodal point-X-point complex at the times of fig.b. (b) The stretching number $a$ has abrupt increases and decreases when the orbit approaches a nodal point at the times indicated in parentheses $\mathrm{A}(0.76), \mathrm{B}(11.5), \mathrm{C}(15.7), \mathrm{D}(25.9), \mathrm{E}(37.0), \mathrm{F}(57.0)$, $\mathrm{G}(68.3), \mathrm{H}(69.5), \mathrm{I}(80.7), \mathrm{J}(82.3)$. 

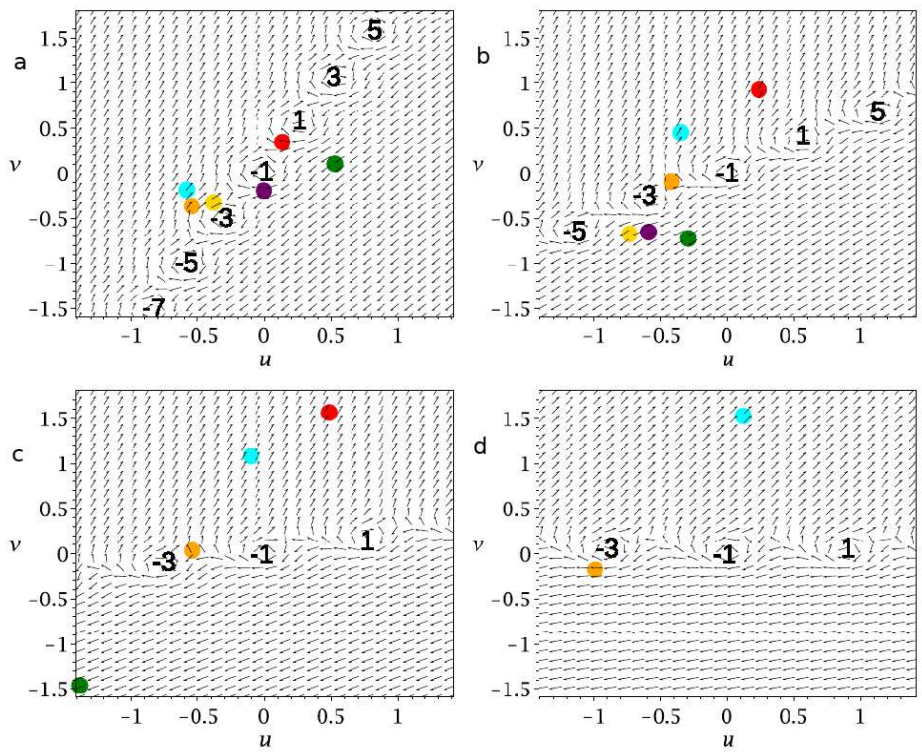

Figure 5. The velocity field in a frame of coordinates centered at the nodal point $k=-1$ at particular times (a) $t=82.660$ (b) $t=82.845$ (c) $t=83.045$ and $t=83.262$ for $c_{2}=2 \times 10^{-5}$. The other nodal points and X-points move around the nodal point $k=-1$. We give also the positions of 6 particles moving along the velocity field that are deflected as they approach some X-points. On the right of the center we have the nodal points $k=1,3,5, \ldots$ and on the left the nodal points $k=-3,-5, \ldots$

\section{Distributions}

If we consider solutions $\Psi$ of the Schrödinger equation and distribute initially the particles according to Born's rule

$$
P_{0}=\left|\Psi_{0}\right|^{2}
$$

at an initial time $t_{0}=0$, then the same relation applies at any later time [27]. However it is of interest to see how this is realized in our model.

As a first example we find the solution of Schrödinger equation for the maximally entangled case (Bell state [6]) where $c_{1}=c_{2}=\sqrt{2} / 2$. In Fig. [6 we see the values of $|\Psi|^{2}$ at successive times. Initially we have two symmetric blobs around the points $x_{0}= \pm 3.54, y_{0}=\mp 2.68$ along the line with an inclination equal to $\theta \simeq-0.65$ radians (red contours). The two blobs have practically disjoint supports $S_{1}$ and $S_{2}$ in $x-y$ 
plane. Then at $t=1$ the blobs have moved closer to the center (green contours) and at $t=2$ they have passed to the other side (blue contours). Their motion is counterclockwise. At $t=3$ (purple contours) and at $t=4$ (dark green contours) they are close to an inclination $\theta \simeq 0.33$ but close to $t=4.5$ the two blobs collide and form a set of several maxima and minima. After this collision the blobs reappear and move around until a second collision takes place and so on.

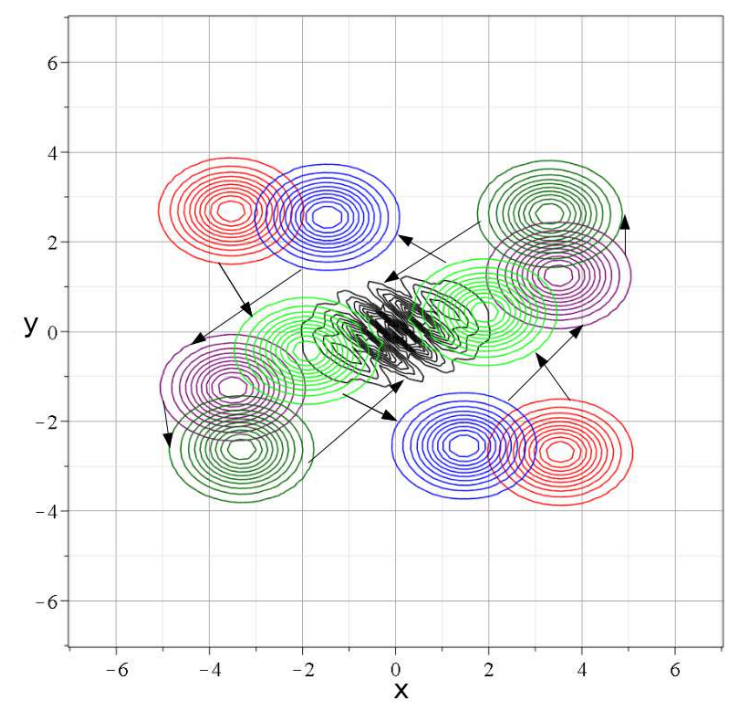

Figure 6. The values of $|\Psi|^{2}$ in the case of maximum entanglement $\left(c_{2}=c_{1}=\right.$ $\sqrt{2} / 2$ ) at successive times: $t=0$ (red), $t=1$ (green), $t=2$ (blue), $t=3$ (purple), $t=3.5$ (dark green) and $t \simeq 4.5$ (black).

If now we populate the areas $S_{1}, S_{2}$ of the blobs with a large number of initial conditions distributed according to the law (16), we can follow in detail how the ensemble of the resulting Bohmian trajectories evolves in time in order to always satisfy the law $P(t)=|\Psi(t)|^{2}$. Figure 7 provides the relevant information. The trajectory points corresponding to initial conditions within the left set $S_{1}$ are shown in blue and those of the initially right set $S_{2}$ are shown in red (Fig. [7 a). The domains covered by the blue and red points collide near $t=4.5$ as shown in Fig. $7 \mathrm{~b}$. After the collision the domains covered by the red and blue points both split, so that the red points form two separate sets and the blue points form also two separate sets. However, the union of all the sets (red+blue) forms two blobs equal to the blobs of 
$|\Psi|^{2}$ (Fig. $7 \mathrm{c}$ ). Later on we have another collision and another splitting of the red and blue sets, but again the total distribution is very close to $|\Psi|^{2}$, as required by the theory (Fig.7 d). Even later we have further splittings but again the total density $P$ remains close to $|\Psi|^{2}$.

This result verifies in a spectacular way that while the relation $P(t)=|\Psi(t)|^{2}$ is satisfied at all later times if $P_{0}=\left|\Psi_{0}\right|^{2}$, the Bohmian trajectories undergo substantial mixing in the configuration space. The mixing is driven by the scattering of the trajectories in various directions, taking place at essentially every collision of the wavefunction blobs corresponding initially to $S_{1}$ and $S_{2}$.

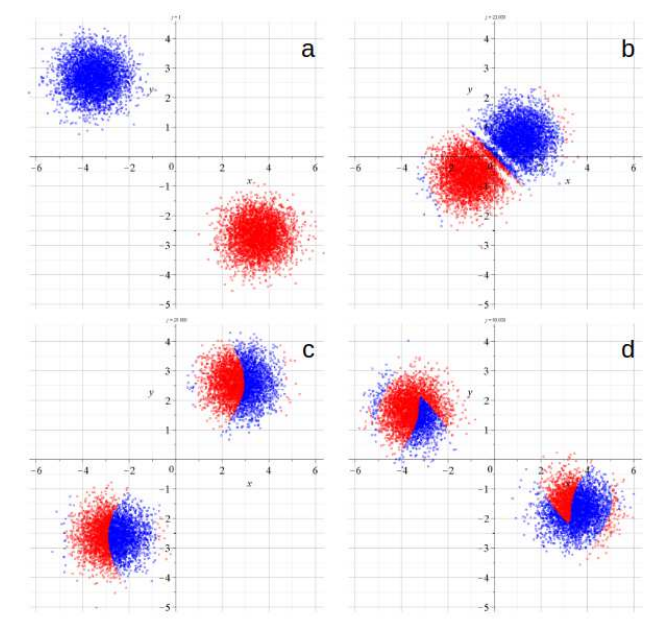

Figure 7. The successive positions of two blobs containing 1000 initial conditions with distribution $P_{0}=\left|\Psi_{0}\right|^{2}$ in the case $c_{1}=c_{2}$ at various times (a) $t=0$, (b) $t=4.5$ when the two blobs collide, (c) $t=5.8$ after the first collision when each blob is split, but their combination gives $P=|\Psi|^{2}$, (d) $t=16$ after the second collision (when again $P=|\Psi|^{2}$ ).

The overall coverage of the space by the red and blue points after a long time is shown in Fig. 8 8 a. This figure was calculated as follows. We covered the central part of the configuration space $(x, y)$ by a large number of cells (a square grid of $360 \times 360$ cells) and we calculated the number of times the trajectories pass through these cells. The total number of initial conditions was 250, distributed according to $P_{0}=\left|\Psi_{0}\right|^{2}$ (approximately) and the total time was $t=1000$. Then we gave to every cell the number of passages of the trajectory through this cell, by means of a color plot. 
In a similar way, in Figs $8 \mathrm{~b}, \mathrm{c}, \mathrm{d}$ we show individual trajectories with initial conditions $\left(x_{0}=-2, y_{0}=2\right),\left(x_{0}=-4, y_{0}=4\right)$ and $\left(x_{0}=-2, y_{0}=0\right)$, calculated for a time $t=7 \times 10^{4}$ in order to have a significant coverage of space. All these figures have regions of large densities around the points $x= \pm 3.8, y= \pm 2.8$ and a very similar distribution of the regions with various colours, including the central regions where we see 4 islands, (surrounded by 8 islands) of smaller density than their surroundings.
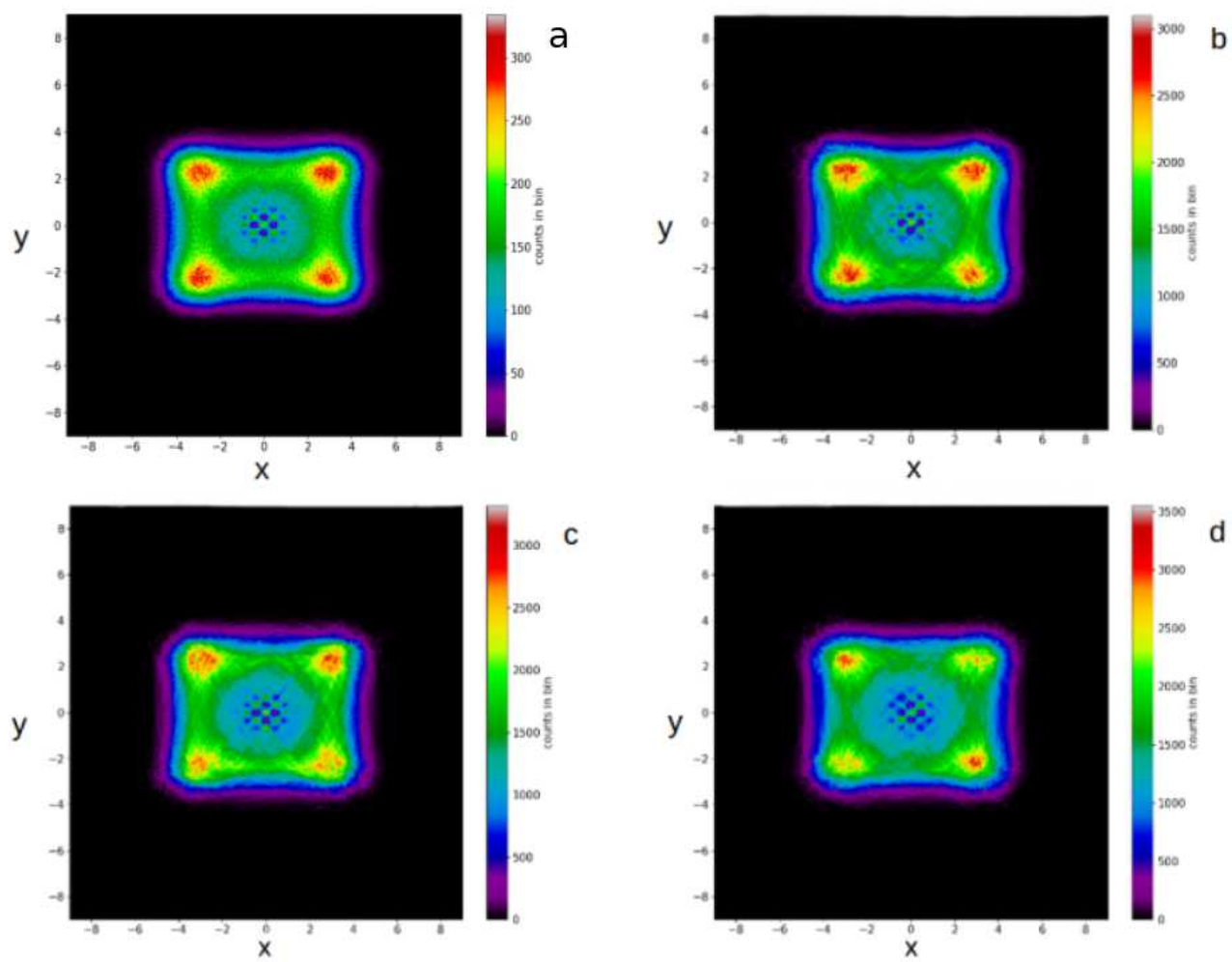

Figure 8. (a) The overall distribution of 250 initial conditions approximating the formula $P_{0}=\left|\Psi_{0}\right|^{2}$ over a time $t=10^{3}$ (limiting distribution). The distributions of the points of particular trajectories for $t$ up to $t=7 \times 10^{4}$, given in Figs (b) for $\left(x_{0}=-2, y_{0}=2\right),(\mathrm{c})$ for $\left(x_{0}=-4, y_{0}=4\right)$ and (d) for $\left(x_{0}=-2, y_{0}=0\right)$ approach very well the limiting distribution.

The fact that these distributions are approximately the same with the total distribution of Fig. 8 a shows that individual trajectories tend to be ergodic. In fact, independently of the initial condition, all individual Bohmian trajectories have the 
same distribution after a long time. This property appears even while the system has no explicit outer boundary. In particlular the trajectory of Fig. 88c and a similar trajectory with initial conditions $(4,-4)$ start at points where $|\Psi|^{2}$ is very small at all times $t$. We notice that the trajectories move to arbitrarily large distances only very rarely. This is indicated by the fact that the outermost parts of Figs 8 are dark purple, which means very small densities in the overall coverage. Nevertheless after a long time the trajectories that start far from the central region cover the space with approximately the same densities as the trajectories starting closer to the center. Similar distributions are found for all the initial conditions of the Bell state (i.e. for $\left.c_{1}=c_{2}=\sqrt{2} / 2\right)$.

As $c_{2}$ decreases down to $c_{2}=0.01$ we found similar results, i.e. four blobs with maximum density but these maxima are closer to the center. E.g. while for $c_{2}=\sqrt{2} / 2 \simeq 0.707$ the maxima are at $x \simeq \pm 3.0, y \simeq \pm 2.4$, for $c_{2}=0.1$ the maxima are at $x \simeq \pm 1.5, y \simeq \pm 1.3$ and for $c_{2}=0.01$ the maxima are at $x \simeq \pm 1.1, y \simeq \pm 0.7$.
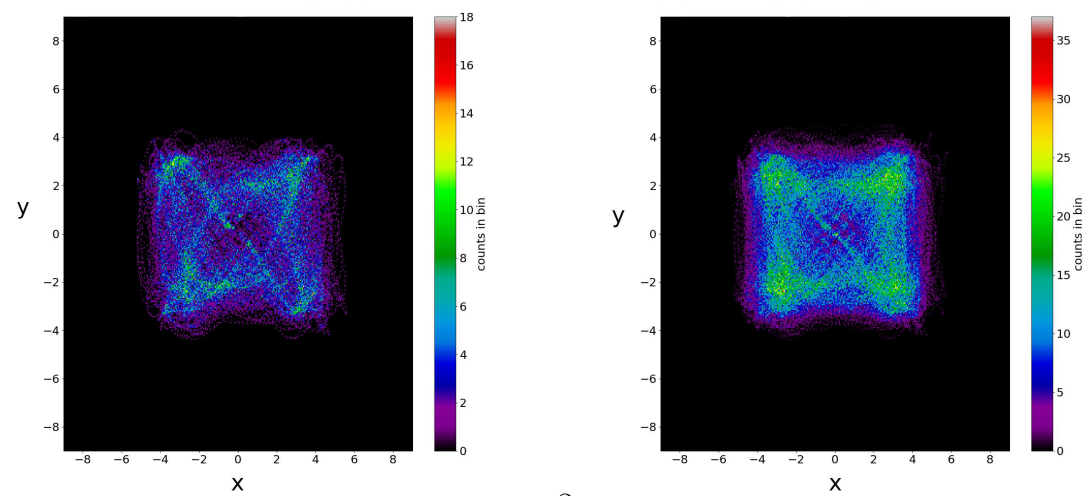

a

$\mathrm{b}$

Figure 9. The distribution of the trajectory with initial conditions $x(0)=$ $-2, y(0)=2$ in the case of maximal entanglement $\left(c_{2} \simeq 0.707\right)$ for: (a) $t=4500$ and (b) $t=15000$ : The trajectory acquires the characteristic form of a limiting distribution in a relatively short time.

Most notably, the time needed by a trajectory to show its ergodic character (i.e. to approach the final average distribution) increases as $c_{2}$ decreases. Namely for $c_{2}=0.707$ we found that the main features of the limiting distribution of Fig. 8 a may be seen already for $t=15000$ (Fig. 9). On the other hand for $c_{2}=0.01$ we need a much longer time in order to approach the limiting distribution. In Fig. 10a 
we see that for $t=15000$ the distribution of the trajectory points does not represent clearly its final form. However, after a time $t=75000$ the distribution approaches satisfactorily its final form (Fig 10b). We have found similar results for several trajectories and various values of $c_{2}$.
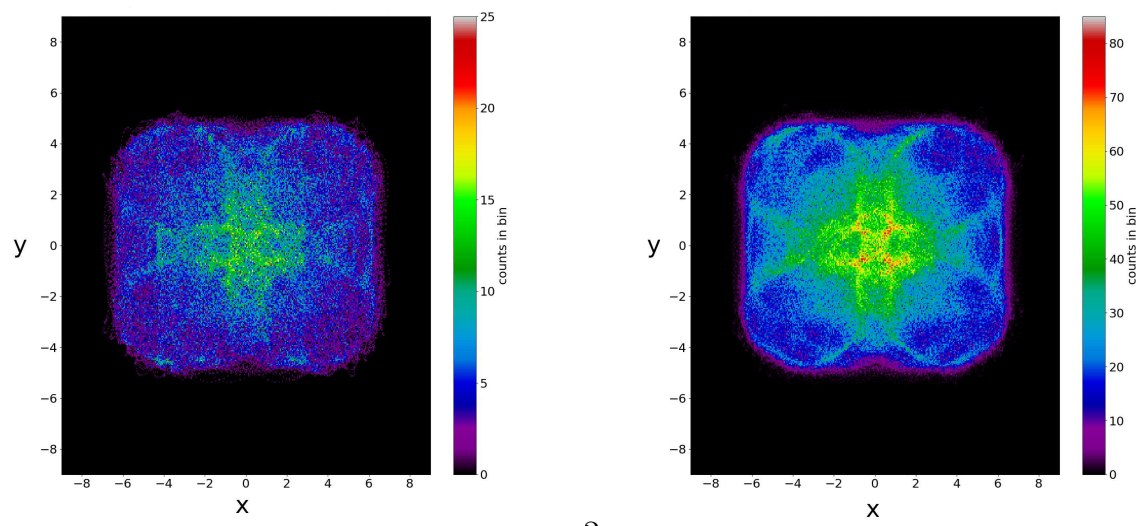

a

$\mathrm{b}$

Figure 10. The distributions of the points of a single Bohmian trajectory in the case of $c_{2}=0.01$, when $x(0)=-2, y(0)=2$ for times (a) $t=15000$ and (b) $t=75000$.

\section{Conclusions}

In this paper we continued our previous work [26] on the effect of quantum entanglement in the evolution of Bohmian trajectories, by studying in more detail and from various perspectives the trajectories of an entangled two-qubit system. This simple bipartite system has the advantage of being simple from a computational point of view. However its unique feature, namely the infinite number of nodal points, provides us with rich information about the effect of entanglement on the Bohmian trajectories.

Our main conclusions are the following:

(i) Chaos is introduced when the moving particles approach the nodal points whose velocities are small in aggreement with the theory of the NPXPCs.

(ii) In our model there are infinite nodal points that fill most of the configuration space. Between the nodal points there are the X-points (unstable hyperbolic 
points of the flow). The distance between any two successive nodal points is the same for any given time $t$. The evolution of the nodal points has slow and fast parts: the fast parts correspond to the almost instantaneous departure of the NPXPCs from infinity along the diagonal lines and the slow parts correspond to the loops of the NPXPCs close to the origin, where the probability of strong scatttering events is high.

(iii) For a very small amount of entanglement the Bohmian trajectories are of Lissajous type close to the unperturbed Lissajous curves (of zero entanglement) for very long times. For a somewhat larger entanglement the Bohmian trajectories are again of Lissajous type for some time but they are displaced with respect to the unperturbed Lissajous figures. After a close approach to a NPXPc these trajectories deviate considerably and form new Lissajous-type curves further away from the initial ones. Finally the trajectories fill chaotically the central region of the configuration space.

(iv) The initial form of the measure of the wavefunction $\left|\Psi_{0}\right|^{2}$ consists of two blobs. These blobs move around and from time to time they collide, but after every collision they form again two moving blobs. If the initial conditions are distributed according to the probability law $P_{0}=\left|\Psi_{0}\right|^{2}$ then after every collision the trajectories of every blob split and follow very different directions. Nevertheless the total distribution produced by the trajectory points always satisfies $P=|\Psi|^{2}$ (Born's rule).

(v) For large entanglements the trajectories are quite chaotic. Their distribution tends to form very characteristic patterns. The individual trajectories, after a sufficiently long time tend to form the same patterns. Thus the trajectories tend to be ergodic although their initial conditions may be quite different. This is an important property that was numerically verified for large enough entanglements. We have numerical indications that the trajectories are ergodic in general, but the time needed for an orbit to show its ergodic character (approach to the average characteristic pattern) is larger for smaller values of entanglement. The time needed for the trajectories to converge to an ergodic behavior increases in general with the decrease of entanglement. Our present computational limitation allowed us to explore the domain $0.01 \leq c_{2} \leq \sqrt{2} / 2$. Further simulations are required to explore the limit $c \rightarrow 0$.

Our main new result, the emergence of ergodicity in entangled systems raises 
new questions about the interplay between chaos and entanglement, which is an important problem in BQM. These first numerical resuts will be the starting point for future studies on this subject addressing questions such as the ergodicity of Bohmian particles a) in the presence of weak entanglement, where we one expects to find initial conditions which lead to ordered trajectories and b) in the special case when Born's rule is not initially satisfied. Finally an open problem is the relation between the degree of entanglement and the time needed to approach ergodicity.

\section{Appendix}

The existence of an infinite number of NPXPCs makes the integration of Bohmian trajectories a quite demanding process. During our calculations we worked with different numerical methods of fixed and adaptive step size and we compared their results. However there were many cases where the calculations were impossible to finish in a reasonable amount of time, due to the extremely small stepsize needed to monitor the evolution of the trajectories (e.g. when the trajectory forms spirals around a moving nodal point) in the case of fixed step methods, or due to the quick violation of error tolerance (smaller than $10^{-6}$ ) in the case of adaptive methods.

In order to observe these tiny details in the first moments of the evolution and to have reliable results for large times (in the case of distributions), we worked in Python 2.7 with the Lsoda adaptive numerical integration scheme [28], with absolute error tolerance $a_{t o l}=10^{-10}$ and relative error tolerance $r_{t o l}=10^{-7}$. Stricter error tolerance values did not alter our results.

\section{Acknowledgments}

The authors thank Dr. C. Efthymiopoulos for his useful comments. This research is supported by the Research Commitee of the Academy of Athens.

\section{References}

[1] Bohm D 1952 Phys. Rev. 85(2) 166

[2] Bohm D 1952 Phys. Rev. 85(2) 180

[3] Holland P R 1995 The quantum theory of motion: an account of the de Broglie-Bohm causal interpretation of quantum mechanics (Cambridge University Press) 
[4] Dürr D and Teufel S 2009 Bohmian Mechanics: The Physics and Mathematics of Quantum Theory (Springer)

[5] Benseny A, Albareda G, Sanz Á S, Mompart J and Oriols X 2014 Eur.Phys.J. D 68 1-42

[6] Nielsen M A and Chuang I L 2004 Quantum Computation and Quantum Information (Cambridge University Press)

[7] Horodecki R, Horodecki P, Horodecki M and Horodecki K 2009 Rev. Mod. Phys. 81865

[8] Frisk H 1997 Phys. Let. A 227 139-142

[9] Bialynicki-Birula I, Bialynicka-Birula Z and Śliwa C 2000 Phys. Rev. A 61032110

[10] Falsaperla P and Fonte G 2003 Phys. Let. A 316 382-390

[11] Wisniacki D A and Pujals E R 2005 Europhys. Lett. 71159

[12] Yang C D and Wei C H 2008 Chaos, Solitons \& Fractals 37988

[13] Chou C C and Wyatt R E 2008 J. Chem. Phys. 128234106

[14] Chou C C and Wyatt R E 2008 J. Chem. Phys. 129124113

[15] Chou C C, Sanz Á S, Miret-Artés S and Wyatt R E 2009 Phys. Rev. Lett. 102250401

[16] Efthymiopoulos C and Contopoulos G 2006 J. Phys. A 391819

[17] Efthymiopoulos C, Kalapotharakos C and Contopoulos G 2007 J. Phys. A 4012945

[18] Contopoulos G, Efthymiopoulos C and Harsoula M 2008 Nonlin. Phen. Compl. Sys. 11107

[19] Efthymiopoulos C, Kalapotharakos C and Contopoulos G 2009 Phys. Rev. E 79(3) 036203

[20] Tzemos A C, Efthymiopoulos C and Contopoulos G 2018 Physical Review E 97042201

[21] Tzemos A C and Contopoulos G 2018 J. Phys. A 51075101

[22] Efthymiopoulos C, Contopoulos G and Tzemos A C 2017 Ann. Fondation L. de Broglie 42 153

[23] Cesa A, Martin J and Struyve W 2016 J. Phys. A 49395301

[24] Zander C and Plastino A 2018 Entropy 20473

[25] Elsayed T A, Mølmer K and Madsen L B 2018 Scientific reports 812704

[26] Tzemos A C, Contopoulos G and Efthymiopoulos C 2019 Phys. Scr. 94105218

[27] Valentini A and Westman H 2005 Proc. R. Soc. A 461 253-272

[28] Linge S and Langtangen H P 2016 Programming for Computations-Python: A Gentle Introduction to Numerical Simulations with Python vol 15 (Springer) 